\title{
SEGUIMIENTO Y EVALUACIÓN EN LA UNED DEL SISTEMA DE PRÁCTICAS DE LOS ALUMNOS EN EMPRESAS
}

\section{Supervision and evaluation of business internships for students in the UNED \\ por}

Article record

$\underline{\text { HTML format }}$

\author{
Belén Ballesteros Velázquez \\ Nuria Manzano Soto \\ Juan Antonio Moriano
}

Ficha del artículo

Formato HTML

\begin{abstract}
In this article we present the basic guidelines proposed for the Guidance, Information and Employment Center (COIE) of the National University for Distance Education (UNED) for the development, management, and evaluation of educational co-operation contracts for field experience in businesses, in which students are requesting to participate with greater frequency. The beginning of this research focuses on the supervision of the development of these programs, and establishing guidelines and design procedures in order to follow-up to improve them. The conclusions presented here are drawn from results that were obtained in the evaluation of internships during the academic course $1998 / 99$ and 1999/00, according to the perspective of the participating enterprises observing student interns, and are organized into a proposal.
\end{abstract}

\section{Keywords}

Evaluation, supervision, practical training, internship

.

\section{Resumen}

En este artículo se presentan las líneas básicas de trabajo iniciadas por el Centro de Orientación, Información y Empleo (COIE) de la UNED para la evaluación de uno de los ámbitos de actividad que resulta cada vez más solicitado por los alumnos: la gestión de convenios de cooperación educativa para la realización de prácticas en empresas. La necesidad de supervisar su desarrollo, establecer mecanismos de seguimiento y, en definitiva, diseñar procedimientos para su mejora, constituyen el punto de partida de esta investigación. Se presentan los resultados conseguidos en la evaluación de prácticas, tanto desde el punto de vista de las empresas como de los alumnos, que han tenido lugar en los cursos académicos 1998/99 y 1999/00, junto con las conclusiones obtenidas y medidas de mejora propuestas.

\section{Descriptores}

Evaluación, seguimiento, prácticas.

\section{INTRODUCCIÓN}

Las prácticas en empresas ofrecen al alumnado de Segundo Ciclo la posibilidad de completar la formación académica que reciben en la Universidad con el desempeño de las tareas propias de una actividad profesional, por medio de estancias breves en empresas u otros centros de trabajo. Por ello, cons- tituyen un elemento clave para la inserción en el mundo laboral, dado que proporcionan una experiencia formativa y profesional que es reconocida y valorada por el propio mercado de trabajo como condición facilitadora para acceder a un determinado puesto de trabajo. En opinión de los empresarios, las relaciones Universidad-empresa en los últimos años son más cercanas y próximas por los distintos planes de colaboración entre 
ambas entidades, en los que se incluyen la oferta de prácticas para alumnos de últimos cursos (Aragón y otros, 1999).

La demanda expresa por parte del alumnado de tales prácticas viene siendo manifestada en diferentes análisis llevados a cabo sobre las necesidades de información y orientación de los universitarios. En este sentido, los resultados muestran las siguientes valoraciones:

- Dentro del área de información y orientación profesional, el alumnado de universidades madrileñas señalan como aspectos más demandados las salidas profesionales seguido de las prácticas en empresas (Sánchez, 1999).

- Los alumnos de nuevos planes de estudio sugieren, entre las propuestas de cambio para la mejora de la calidad en la Universidad, una formación conectada con el mundo del trabajo (Cajide y otros, 1997).

- Las expectativas y deseos de los alumnos se centran en las actividades de orientación profesional, entre las cuales se incluyen acciones para el conocimiento del mundo laboral y programas de desarrollo de habilidades para la profesión (Ausín y otros, 1997).

En nuestro contexto específico, los alumnos de la UNED indicaron la necesidad de favorecer actuaciones concretas a favor de la inserción laboral, entre las cuales figuran las prácticas en empresas (Sebastián y otras, 1996).

Desde el punto de vista formal, la realización de estas prácticas exige la firma de un convenio de cooperación educativa entre nuestra Universidad y la empresa o entidad que acoge al alumno, tarea que actualmente están desarrollando los COIEs acogiéndose a la normativa establecida por el Real Decreto 1497/1981, de 19 de junio y actualizado por el Real Decreto 1845/1994, de 9 de septiembre, por la cual se regulan los aspectos que se detallan en el siguiente apartado.

\section{LAS PRÁCTICAS A TRAVÉS DE CONVENIOS DE COOPERACIÓN EDUCATIVA ESTABLECIDOS EN- TRE LA UNIVERSIDAD Y LA EM- PRESA}

Las prácticas en empresas son estancias en centros de trabajo, privados o públicos, que permiten al universitario completar su formación académica a través del ejercicio, bajo tutela, de las competencias propias de su área profesional para la cual están siendo preparados.

Para ello, la Universidad suscribe un convenio con el centro interesado en la admisión de alumnos en prácticas, siguiendo la normativa vigente al respecto que establece los requisitos y condiciones que se detallan a continuación:

- Pueden realizar prácticas únicamente los alumnos que ya hayan superado la mitad de créditos de su carrera (planes nuevos) o estén en los dos últimos cursos (planes antiguos).

- Se nombrará un tutor de prácticas por parte de la Universidad y otro por parte de la empresa.

- La duración de las prácticas no excederá el 50\% de horas lectivas de un curso académico, siendo establecida su duración máxima en 500 horas.

- Durante el período de prácticas el alumno debe estar cubierto por un seguro que, para el caso de los menores de 28 años, se corresponde con el seguro escolar. Para alumnos que superen esta edad, se establece la necesidad de suscribir otro tipo de seguro, bien por parte del alumno, la empresa o la Universidad.

- La realización de las prácticas no conlleva ninguna obligación contractual por parte de la empresa.

- El alumno en prácticas no puede ser contratado por la empresa hasta que éste finalice sus prácticas o se impugne el convenio. 
- Se contempla la posibilidad de remunerar la actividad desarrollada por el alumno en el centro de trabajo a través de la dotación de una beca.

El desarrollo de las prácticas ofrece importantes ventajas para el alumno, tanto desde el punto de vista académico como para su inserción laboral. Los alumnos consiguen una formación más sólida y completa, puesto que la realización de una práctica implica una serie de oportunidades y contactos que les permiten la adquisición de conocimientos significativos al conectar la teoría con la práctica real del desempeño profesional. Las prácticas, por tanto, ofrecen un espacio para el desarrollo de competencias, entendidas no sólo como conocimientos teóricos sino también como destrezas y habilidades necesarias para la realización eficaz de tareas propias de la profesión.

Por otra parte, las prácticas constituyen una respuesta a uno de los problemas principales que encuentra el universitario para el acceso al mercado laboral. Las entidades y empresas que ofertan empleo en el sector privado suelen demandar un tipo, más o menos prologado, de experiencia profesional. Ya queda lejos una etapa en la que la obtención de un título universitario abría consigo la puerta al mercado laboral. En la situación actual, los estudios universitarios no garantizan la consecución de un puesto de trabajo. No se exige sólo un título, sino también otra serie de conocimientos y experiencias profesionales para la obtención de un empleo, incluso si es el primero. El recién titulado se encuentra en una especie de bucle: "quiero un trabajo, pero me piden experiencia que no tengo. Y, por tanto, no llego a adquirir experiencia porque no consigo trabajo". En este sentido, la realización de un período de práctica en empresas durante el transcurso de la carrera universitaria, como estrategia para el aumento de la empleabilidad, supone una vía de solución a esta dificultad inicial que tanto acusan en la actualidad los recién titulados, principalmente.
Otras de las ventajas que suponen las prácticas para la inserción profesional y desarrollo académico del alumno, siguiendo las ideas expuestas por Ballesteros, Guillamón, Manzano, Moriano y Palací (2001), se sintetizan en las siguientes:

- Las prácticas plantean situaciones difíciles de resolver que exigen el desarrollo de competencias profesionales para su respuesta.

- Facilitan el reajuste de expectativas del alumno frente a la situación real que presenta el mundo laboral.

- Ayudan a detectar puntos fuertes y débiles en relación con el desempeño de tareas profesionales, favoreciendo así la toma de decisiones para la reorientación de la carrera.

- Amplían el número de conocidos, (alumnos de nueva titulación, empleadores, etc.)

- Pueden llegar a constituir una forma de reclutamiento laboral. El período de prácticas supone una forma de conocimiento de posible trabajadores que puedan incorporarse a la empresa, por lo que las prácticas, tal y como veremos posteriormente, son una forma de selección de profesional. Este hecho es positivo tanto para el alumno como para las empresas.

Bien es cierto que el desarrollo de las prácticas puede ocasionar también una serie desventajas para al alumno, sobre todo en aquellos casos en los que la gestión y desarrollo de las mismas no están bien organizados de acuerdo con unas metas formativas. En este sentido, los mismos autores advierten del peligro de asimilar las tareas de prácticas con la realización de trabajos que poco o nada tienen que ver con las funciones profesionales para las cuales se está preparando el alumno en la Universidad. La ausencia, en ocasiones, de un plan efectivo de seguimiento y tutorización desfavorecen este valor formativo del período de prácticas. Asimismo, el alumno deberá valorar en todo momento las consecuencias que le supone la dedicación de tiempo en la empresa, pues es 
evidente que ello le restará tiempo a sus estudios.

Con todo, la necesidad de evaluar la gestión y desarrollo de las prácticas resulta evidente para los centros responsables de esta función, en tanto que la evaluación supone la acumulación de la información necesaria para la toma de decisiones conducentes a la mejora de esta actividad.

\section{LA EVALUACIÓN COMO ES- TRATEGIA DE MEJORA EN LA GESTIÓN Y DESARROLLO DE PRÁCTICAS}

La evaluación en el contexto educativo resulta un proceso necesario para la implantación de planes de mejora. La adaptación de cualquier centro a un entorno cambiante no se lleva a cabo de una manera automática, sino que requiere un análisis y reflexión que oriente el diseño de líneas futuras de actividad (López Rupérez, 2000). El carácter formativo de la evaluación se relaciona de manera reiterada con la calidad de los programas e instituciones. Sin embargo, el concepto de calidad no adopta un enfoque único, sino que ha de ser entendido en función de intereses y situaciones diversas. En este sentido, González Ramírez (2000) destaca una serie de rasgos característicos que ponen de manifiesto la imposibilidad de concebir la calidad de una forma unívoca: su valor contextual, la necesidad de valorarse de forma plural, su carácter diacrónico y la exigencia de acudir al mundo de los valores y sus consecuencias.

Desde nuestra posición, como personal que colabora en el COIE de la UNED, entendemos la calidad de las prácticas en función de su valor académico y profesional. Para ello, estimamos necesario el diseño de un procedimiento riguroso para su supervisión, con el fin de asegurar que las tareas que nuestros alumnos desempeñan en las entidades con las que se haya firmado un convenio sean plenamente formativas y se ajusten única $\mathrm{y}$ exclusivamente al objetivo de incrementar la formación en el trabajo.

El Plan Nacional de Evaluación de la Calidad adoptado por las universidades desde 1995 se orienta fundamentalmente a la evaluación de las titulaciones, sin que encuentren dentro de su marco de actuación las distintas actividades que son desarrolladas en otros centros distintos a las escuelas o facultades. Sin embargo, la evaluación de las prácticas constituye un indicador sobre el desarrollo de la enseñanza en cuanto que incluye el análisis de acuerdos y convenios específicos con empresas, organismos y entidades en relación con la titulación evaluada, dentro del apartado referido a la metodología docente, (UNED, 2000). Por tanto, la evaluación de las prácticas constituye asimismo una información transversal para las distintas facultades y escuelas, si bien es cierto que actualmente no hay establecida una política de colaboración entre el profesorado universitario y el COIE para la gestión de convenios de colaboración educativa, recayendo esta tarea en el COIE en exclusividad.

En el caso específico de nuestra Universidad la evaluación de las prácticas debe considerar las peculiaridades propias que adopta la organización del COIE de la UNED. En esta organización cabe distinguir entre COIE de la Sede Central, ubicado en Madrid, y COIEs en los Centros Asociados, distribuidos por la geografía española a través de veinticinco centros. Desde la Sede Central se coordina la actuación de los COIEs en Centros Asociados, atendiéndose igualmente a las demandas de información, orientación y gestión de prácticas y empleo de los alumnos en Madrid y en otros centros que no disponen de COIE. Los COIEs en Centros Asociados tienen acotado su marco de actuación a su ámbito geográfico específico en el que están situados.

La presentación del proyecto de evaluación y seguimiento del sistema de prácticas en 
empresas de la UNED fue diseñado inicialmente en el COIE de la Sede Central, siendo presentado posteriormente a los Centros Asociados que de forma voluntaria decidieron su participación o no en esta tarea ${ }^{[1]}$. Las líneas generales que definían el proyecto de evaluación se presentan en los siguientes apartados referidos a la definición de objetivos y a la metodología propuesta.

\subsection{Objetivos del plan de evaluación y seguimiento del sistema de prácticas en empresas de la UNED}

Desde el COIE de la UNED se plantea la necesidad de un estudio evaluativo con el fin de establecer en último término un sistema de actuación y seguimiento en el desarrollo de las prácticas de nuestros alumnos que garantice el valor formativo de las mismas. Para ello, se pretende alcanzar la información necesaria para responder a las siguientes cuestiones:

- Verificar el grado de ajuste de las prácticas realizadas en los últimos cursos académicos a la normativa vigente.

- Comprobar el valor formativo de las prácticas, valorando los siguientes aspectos: formación en competencias profesionales, formación en habilidades sociales propias del trabajo en las organizaciones (comunicación, negociación, manejo de conflictos, etc...).
- Valorar la incidencia de las prácticas en el desarrollo de la carrera y, más concretamente, en la inserción laboral de los alumnos.

- Determinar la existencia de posibles diferencias en el desarrollo de las prácticas en función de las distintas titulaciones.

- Determinar el perfil del alumno que realiza prácticas en empresas.

- Describir las características de las empresas o entidades que con más frecuencia ofrecen oportunidades de prácticas por medio de la firma de convenios de cooperación educativa de nuestra Universidad.

- Mejorar el actual sistema de desarrollo y supervisión de prácticas en empresas.

\subsection{Diseño de la evaluación}

Para la consecución de la información necesaria para responder a las cuestiones que se analizan y diseñar posteriormente la líneas de actuación y mejora del sistema de prácticas, se ha optado en un primer momento por un estudio de tipo descriptivo siguiendo la metodología basada en encuestas. Para ello se ha elaborado cuestionarios específicos dirigidos a los tres colectivos implicados en la gestión y desarrollo de las prácticas: alumnos, empresas y COIEs de los Centros Asociados de la UNED y Sede Central propiamente, tal como se expone en el cuadro que sigue:

Tabla 1.- Enfoque metodológico, muestra e instrumentación.

\begin{tabular}{|c|c|c|}
\hline Enfoque & Muestra & Instrumentos \\
\hline \multirow{3}{*}{$\begin{array}{c}\text { Estudio tipo } \\
\text { encuesta }\end{array}$} & $\begin{array}{c}\text { Alumnos que hayan realizado prácticas en em- } \\
\text { presas a través de un Convenio de Cooperación } \\
\text { Educativa }\end{array}$ & $\begin{array}{c}\text { Cuestionario de Evaluación de las } \\
\text { Prácticas (Alumnos) }\end{array}$ \\
\cline { 2 - 3 } & $\begin{array}{c}\text { Empresas y entidades que hayan firmado Conve- } \\
\text { nios de Cooperación Educativa con la UNED }\end{array}$ & $\begin{array}{c}\text { Cuestionario de Evaluación de las } \\
\text { Prácticas (Empresas) }\end{array}$ \\
\cline { 2 - 3 } & $\begin{array}{c}\text { Responsables de los COIEs en los Centros Aso- } \\
\text { ciados y en la Sede Central }\end{array}$ & $\begin{array}{c}\text { Cuestionario de Evaluación de las } \\
\text { Prácticas (Centros Asociados) }\end{array}$ \\
\hline
\end{tabular}

La muestra total corresponde a la totalidad de alumnos y empresas que han participado en los convenios de cooperación educativa durante los cursos 1998/99 y 1999/00.
Habiendo concluido las prácticas en la fecha de inicio de la recogida de datos.

La población total, coincidente con la muestra invitada, está conformada por formada por 179 alumnos y $59^{[2]}$ empresas, de 
Ballesteros Velázquez, B., Manzano Soto, N. y Moriano, J. A. (2001). Seguimiento y evaluación en la UNED del sistema de prácticas de los alumnos en empresas. RELIEVE, v. 7, n. 1, p. 3-21.

http://www.uv.es/RELIEVE/v7n1/RELIEVEv7n1_1.htm

los cuales se han obtenido respuestas de 108 alumnos y 36 empresas, representando con ello el $60.33 \%$ y $61.01 \%$. La participación, por tanto, es muy elevada, aunque el reducido tamaño de la población original limitarán la generalización de los resultados obtenidos en ambos colectivos. Por otra parte, se obtuvo respuesta de todos los responsables de los COIEs en Centros, si bien los resultados re- cabados de este colectivo no van a ser tratados en este artículo. Por esta misma razón omitimos la descripción del cuestionario aplicado en este grupo.

Los cuestionarios correspondientes a alumnos y empresas incluyen las siguientes dimensiones de estudio.

Tabla 2. Elementos de estudio incluidos en los cuestionarios

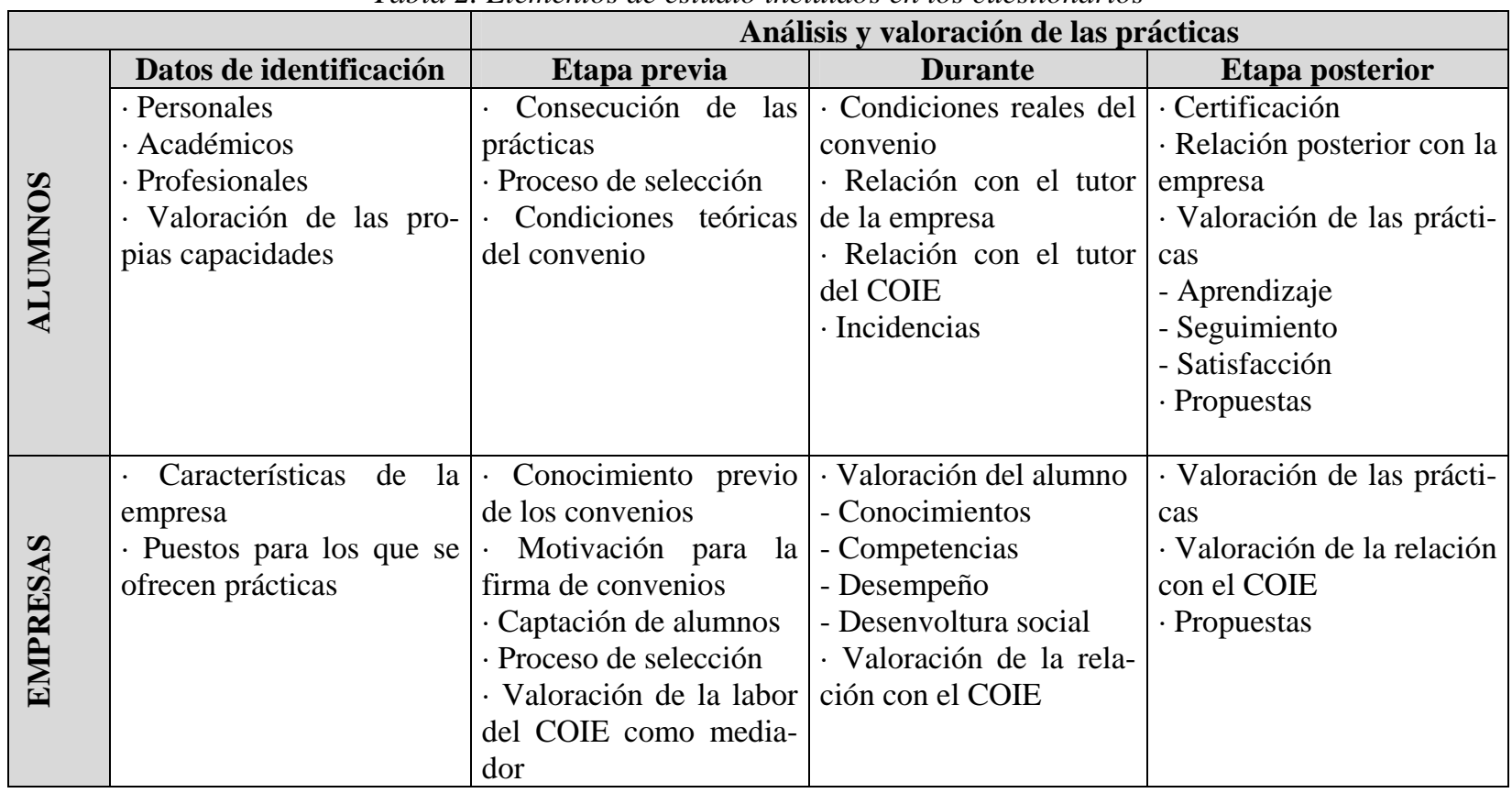

\section{RESULTADOS.}

\subsection{Datos descriptivos.}

\subsubsection{De identificación.}

- Perfil del alumno de prácticas de la UNED.

La edad media de los alumnos oscila entre los 25 y 28 años, predominando las mujeres que realizan prácticas frente a los hombres y, principalmente, los estudiantes que cursan las carreras de Psicología, Económicas / Empresariales, Derecho y Educación. La procedencia geográfica de los alumnos es diversa, resaltando Toledo y Madrid. En cuanto a su formación complementaria, una cuarta parte ha realizado una segunda carrera, dos terce- ras partes tienen conocimientos de informática y tres cuartas partes, dominan otra lengua, generalmente el inglés. Con respecto a su experiencia profesional, la mitad de ellos ya tienen experiencia previa aunque no relativa a sus estudios universitarios.

- Características generales y demandas de las empresas de prácticas.

La mayor parte de las empresas que han ofertado prácticas pertenecen al sector privado (61\%), si bien están aumentando cada vez más los organismos públicos que acogen alumnos en prácticas mediante el COIE (38\%). Más de un $86 \%$ son empresas grandes y medianas, y ubicadas en diversas zonas geográficas de España, aunque predomina Madrid. 
Ballesteros Velázquez, B., Manzano Soto, N. y Moriano, J. A. (2001). Seguimiento y evaluación en la UNED del sistema de prácticas de los alumnos en empresas. RELIEVE, v. 7, n. 1, p. 3-21.

http://www.uv.es/RELIEVE/v7n1/RELIEVEv7n1_1.htm

La principal motivación de la empresa para acoger a alumnos en prácticas es realizar una labor social al posibilitar la formación práctica de los alumnos universitarios. Las empresas no se preocupan exclusivamente de velar por sus beneficios, sino que se involucran en fines más altruistas, procurando devolver a la sociedad los beneficios que de ella obtiene por medio de su contribución a la formación de futuros profesionales. En segundo lugar, la empresas reconocen que

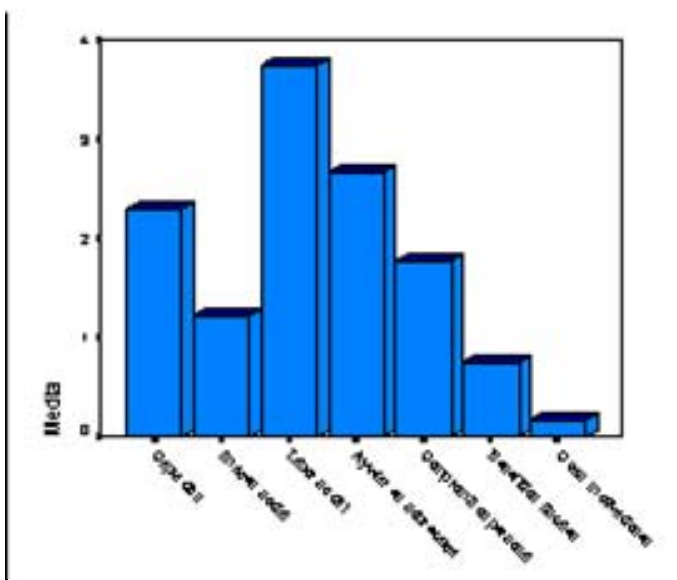

Gráfico 1: Razones para admitir alumnos en prácticas

Las carreras universitarias más demandas por las empresas para la realización de las prácticas son las siguientes: psicología, eco- incorporan alumnos en prácticas para ayudar en momentos de sobrecarga de trabajo, es decir, aprovechar la estancia del alumno como mano de obra barata. Esto supone un aviso para los COIEs en el sentido de que se debe llevar un mayor control sobre cuáles son las tareas que el alumno realiza en la empresa y no permitir que las empresas aprovechen las prácticas como una forma de relación laboral encubierta.
Estadísticos

\begin{tabular}{|l|r|r|}
\hline & \multicolumn{1}{|c|}{ Medi } & Desu. \\
\hline Captació & 2,71 & 1,57 \\
Imagen & 1,30 & 1,05 \\
Labor & 3,52 & 1,48 \\
Ayudar en & 2,88 & 1,61 \\
Compromis & 1,76 & 1,28 \\
personale &, 85 &, 76 \\
Eeneficios & 19 &, 79 \\
Otras & \\
\hline
\end{tabular}

nómicas, empresariales, ingeniería industrial, e informática.

Gráfico 2: Razones para admitir alumnos en prácticas

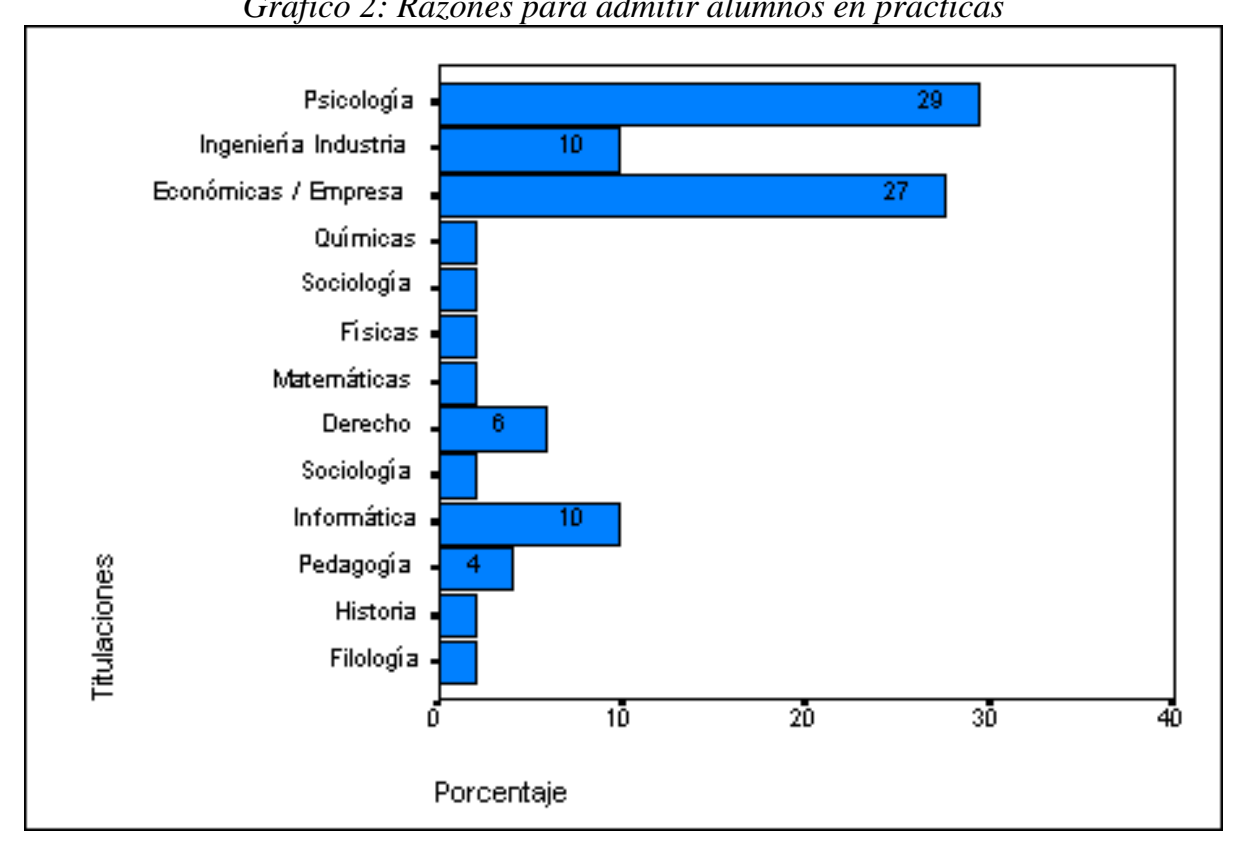


Respecto a los idiomas necesarios para la realización de las prácticas el 36’1\% de las empresas requieren que los alumnos dominen algún idioma. El idioma más demandado sigue siendo el inglés (52'6\%), también se aprecia un aumento del alemán (21’1\%) y un descenso del francés (10’5\%).

Los conocimientos de informática se están volviendo esenciales, especialmente para los universitarios que se ocupan de puestos más especializados donde continuamente deben utilizar el ordenador y las nuevas tecnologías de la información. Según nuestros resultados, en un 72 '2\% de los puestos ofertados en prácticas se requiere conocimientos de informática. Sin embargo, sólo en el 13'8\% casos se exige conocimientos a nivel programador, por tanto, la mayoría las empresas o instituciones necesitan alumnos en prácticas que sepan trabajar con las nuevas tecnologías a nivel usuario.

\subsubsection{Descripción de las prácticas.}

- Desde el punto de vista del alumno.

En la mayoría de los convenios la forma de contacto entre el alumno y la empresa fue a través del COIE que actúa como mediador, difundiendo las prácticas y realizando la preselección de los alumnos que se ajustan al perfil profesional de los puestos ofertados. Dichas ofertas proceden mayoritariamente de grandes empresas que basan el proceso de selección de los alumnos en entrevistas de trabajo y valoración del currículum vitae.

En cuanto a las funciones encomendadas a los alumnos en las prácticas y las funciones reales que han desarrollado, nos hemos basado en las respuestas a dos preguntas abiertas en las que se les solicitaba a los alumnos que expusieran tales funciones. Obviamente, existe una diversidad de tareas según el enfoque de la práctica y de la carrera de la que procede el alumno, no obstante puede apreciarse un predominio de tareas relacionadas con los ámbitos psicológico, educativo y empresarial. En términos generales, puede afirmarse que se da una correspondencia entre las funciones propuestas y las desarrolladas en la práctica real. En los casos en los que desempeñan algunas tareas fuera de las planificadas, éstas suelen ser de carácter administrativo o de servicio, aunque son los menos casos.

Con respecto al horario seguido en las jornadas de prácticas, oscila entorno a cuatro horas y media según el horario acordado y cinco horas según el horario real dicho por los alumnos, llegando la duración total de las prácticas aproximadamente a las 300h en la mayoría de convenios. Dichas prácticas reciben escasa ayuda económica consistiendo, en esos casos, en concepto de remuneración de prácticas, dietas o transporte.

En la mayoría de los casos los alumnos reciben la certificación de las prácticas realizadas tras su finalización y a una cuarta parte de éstos se les propone un contrato laboral, aunque sólo se firman la mitad de estas propuestas.

- Desde el punto de vista de la empresa.

Las prácticas suponen un excelente medio de captación y selección para las empresas. El desarrollo de prácticas permite la observación de posibles candidatos en el entorno real de trabajo. Una selección basada en la observación sistemática de aspirantes es indudablemente más fiable y valida que otra basada en la pericia de los técnicos de selección en el pronóstico mediante las técnicas existentes (entrevistas, tests, etc.) del futuro rendimiento de los aspirantes.

Las empresas contactan con los alumnos de la UNED para la realización de prácticas a través del propio COIE (44’2\%), por iniciativa del propio alumno (44'2\%) y por oferta que publica la propia empresa (8 $3 \%$ ). Con estos resultados, podemos comprobar la importancia de contar con una herramienta informática que permita tener actualizada una base de currículos de los alumnos para poder responder de forma inmediata a la demanda que realizan las empresas. Por otra parte, se observa la importancia de la mediación del COIE y de la propia iniciativa del alumno para realizar una búsqueda activa de sus prácticas, sugerencia que desde este centro hacemos a nuestro alumnado, pues se genera más oportunidades y sirve como “carta de presentación” del alumno ante la empresa.

Por otro lado, las empresas utilizan como forma de selección de alumnos la preselección 
Ballesteros Velázquez, B., Manzano Soto, N. y Moriano, J. A. (2001). Seguimiento y evaluación en la UNED del sistema de prácticas de los alumnos en empresas. RELIEVE, v. 7, n. 1, p. 3-21.

http://www.uv.es/RELIEVE/v7n1/RELIEVEv7n1_1.htm

ofrecida por el COIE (58’3\%) y la referencia directa (44'4\%). A la vista de estos datos, se puede afirmar la eficacia del COIE en la adecuación persona/puesto y la importancia que tiene la iniciativa de los alumnos que se acercan a las empresas en busca de la firma de un convenio de cooperación educativa, ya que son directamente seleccionados para esas prácticas.

El convenio de cooperación educativa establece que la empresa debe asignar un tutor que supervise la realización de la práctica. La persona asignada por la empresa para tutelar la labor que realiza el alumno en prácticas suele ser el responsable de ese puesto (57\%) o una persona del departamento de Recursos Humanos (34\%). Consideramos este dato positivo porque garantiza que el tutor conoce el puesto en profundidad.

Los departamentos en los que los alumnos realizan las prácticas son diversos: Recursos Humanos, Administración, Marketing, Informática, Investigación y Desarrollo, Calidad y Medio Ambiente, etc. De esta forma, los pues-

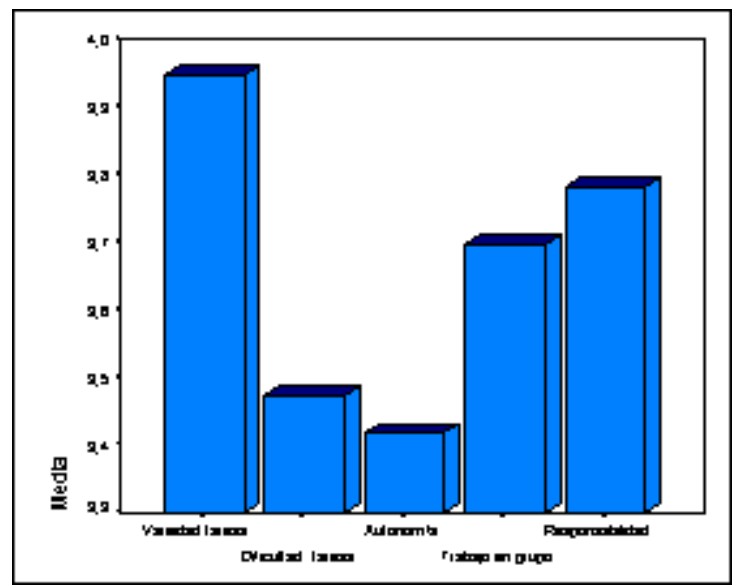

Gráfico 3: Características del puesto

Según la empresa, el horario de prácticas suele ser de media jornada (4 o 5 horas), por la mañana y de lunes a viernes, y tienen una duración total promedio de 356 horas. Sólo el 32\% de las empresas extienden las prácticas hasta su máxima duración, es decir, las 500 horas por curso académico.

En cuanto a la ayuda económica, existe cierta controversia debida a que no se regula la cantidad de ayuda que deberá asignar la empresa al tos desempeñados por los alumnos han sido variados: auxiliar de selección, asistente de tarifas y facturación, ayudante técnico, consultor en prácticas, becario, alumno en prácticas, etc.

Las características del puesto son claves para que el alumno realmente pueda adquirir nuevas competencias profesionales y aplicar los conocimientos que ha adquirido durante su formación académica. Según una escala de 1 a 5 (1 mínimo y 5 máximo) la empresa considera que éstas son las dimensiones que caracterizan el puesto en prácticas: alta variedad en las tareas, dificultad normal, autonomía normal, posibilidad de trabajar en grupo y adecuada responsabilidad. A la vista de estos resultados, se puede afirmar que las tareas y funciones que realiza el alumno en la empresa son adecuadas para su desarrollo profesional. Por tanto, podemos desterrar el estereotipo de que los alumnos en prácticas sólo desarrollan tareas monótonas que nadie quiere realizar en la empresa.

\section{Estadísticos descriptivos}

\begin{tabular}{|l|r|r|}
\hline & \multicolumn{1}{|c|}{ Media } & Desv. típ. \\
\hline Variedad de las tareas & 3,94 & 1,09 \\
Dificultad de las tareas & 3,47 & 1,06 \\
Autonomía & 3,42 & 1,18 \\
Trabajo en grupo & 3,69 & 1,24 \\
Responsabilidad & 3,78 & 1,22 \\
\hline
\end{tabular}

alumno [3]. Por ello, el COIE de la UNED no impone a la empresa una cantidad sino que permite que sea ella misma quien decida si ofrece una ayuda al estudiante y la cuantía de esa ayuda. Según los resultados obtenidos, la ayuda económica que recibe mensualmente el alumno de la empresa oscila entre las 20.000 pts y las 70.000 pts. Sin embargo, es evidente que este tema resulta peliagudo porque el 58'3\% de las empresas encuestadas no han contestado a la pregunta. Por 
Ballesteros Velázquez, B., Manzano Soto, N. y Moriano, J. A. (2001). Seguimiento y evaluación en la UNED del sistema de prácticas de los alumnos en empresas. RELIEVE, v. 7, n. 1, p. 3-21.

http://www.uv.es/RELIEVE/v7n1/RELIEVEv7n1_1.htm

otra parte, el 19`4\% reconoce que no dan ninguna ayuda a los alumnos en prácticas. Una de las razones que esgrimen es que la formación práctica que están adquiriendo los alumnos es de por si suficientemente valiosa. En definitiva, la mayoría de las empresas no entregan bolsa o ayuda al estudio, y aquellas que si lo hacen, lo consideran más como una remuneración por el trabajo realizado por el alumno, que como una ayuda para dietas y transporte.

Entre los beneficios que el alumno puede encontrar en las prácticas resalta la formación, ya que el 94’3\% de ellos reciben algún tipo de formación durante su estancia en la empresa. Las fórmulas varían entre: demostraciones en el puesto de trabajo (51'9\%), consulta de manuales propios de la empresa (32'7\%) y, en menor medida, asistencia a conferencias en la propia empresa (15’ 4\%).

Al término de las prácticas, el 34’3\% de empresas ofrecen un contrato al alumno. Por tanto, esto confirma que las prácticas se están convirtiendo en un de los canales más importantes para acceder a un primer empleo acorde con la formación adquirida durante la carrera universitaria. El tipo de contrato que se ofrece es indefinido en un $30{ }^{\prime} 4 \%$ de los casos, aunque varían entre contratos eventuales (21'7\%), de obra (21'7\%), en

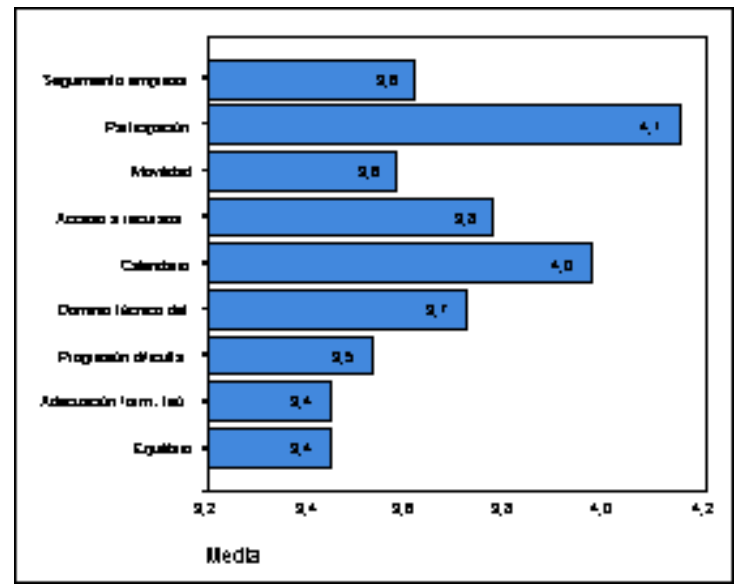

Gráfico 4: Valoración del desarrollo de las prácticas

Respecto a la valoración que ofrecen tras finalizar las prácticas, se observa que valoran positivamente las prácticas en cualquiera de los aspectos señalados. Sin embargo, no prácticas (8’7\%), a tiempo parcial (8’7) y de interinidad (8'7\%).

Se puede apreciar cómo las prácticas se han convertido en un valioso sistema de captación y reclutamiento, ya que el 71 ' $4 \%$ de las empresas archivan en su base de datos los currículos de los alumno que han estado en prácticas. Además, la red de contactos del alumno se amplía facilitándole posteriores relaciones en el mercado laboral. Esta afirmación puede corroborarse, puesto que el 61'8\% de las empresas siguen manteniendo el contacto con el alumno después de las prácticas.

\subsubsection{Valoración de las prácticas y conse- cuencias.}

- El alumno: durante y después de las prácticas.

El alumno ha valorado -en una escala de 1 a 5algunos aspectos de las prácticas tanto durante la realización de las mismas como al finalizarlas. En general los alumnos no presentan ninguna queja o incidencia en el desarrollo de las prácticas. No obstante, en los casos en los que sí se señalan incidencias, suelen estar referidas a la falta de tutorización por parte de la UNED, además de otras cuestiones organizativas en la planificación de las tareas.

Estadisticos descriptivos

\begin{tabular}{|l|r|r|}
\hline & \multicolumn{1}{|c|}{ Media } & Desv típ. \\
\hline Seguiritiento CoIE & 2,65 & 1,61 \\
Seguiniento empresa & 3,57 & 1,48 \\
Participación & 4,15 & 1,17 \\
Movildad & 3,55 & 1,38 \\
Acceso a recursos & 3,75 & 1,29 \\
Calendario & 3,99 & 1,19 \\
Dominio técnico del tutor & 3,71 & 1,49 \\
Progresión dificultad & 3,53 & 1,34 \\
Adecuación form. teórica & 3,44 & 1,29 \\
Equilbrio & 3,44 & 1,27 \\
\hline
\end{tabular}

consideran con el mismo entusiasmo que las prácticas aumenten su empleabilidad aunque existe cierta variabilidad en las respuestas de esta cuestión. 
Ballesteros Velázquez, B., Manzano Soto, N. y Moriano, J. A. (2001). Seguimiento y evaluación en la UNED del sistema de prácticas de los alumnos en empresas. RELIEVE, v. 7, n. 1, p. 3-21. http://www.uv.es/RELIEVE/v7n1/RELIEVEv7n1_1.htm

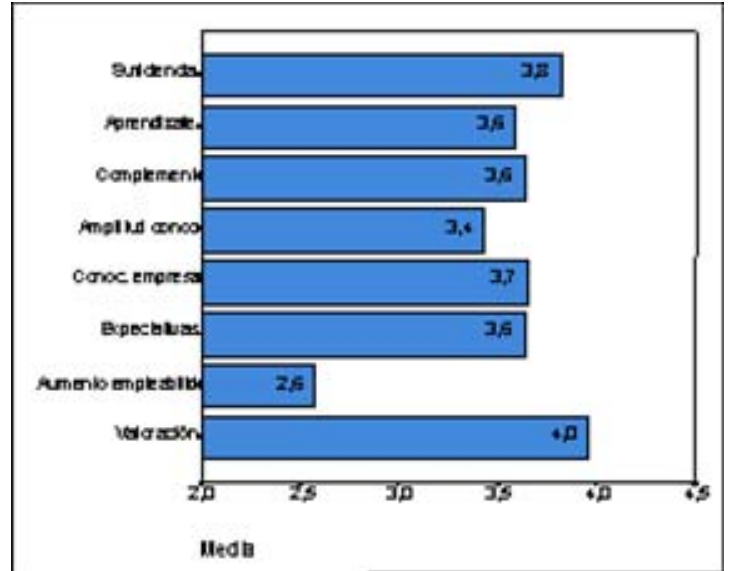

Gráfico 5: Valoración tras la realización de las prácticas

\section{- La empresa.}

En el siguiente punto, se muestra la valoración ofrecida por la empresa respecto a diferentes aspectos relativos al desempeño del alumno en su periodo de prácticas. Se presenta un elenco de las capacidades y hábitos importantes en un entorno laboral, y la valoración dada tanto por el alumno como por la empresa. De esta forma, se ilustran las coincidencias y discrepancias existentes entre ambas partes.

\subsection{Valoración de las capacidades y hábitos de los alumnos, por parte de la empresa $y$ del alumno}

Uno de los puntos que estimamos de especial interés para el desarrollo de actividades formativas y orientadoras a través del COIE, es el referido al estudio de las capacidades del alumnos en
Estadísticos descriptivos

\begin{tabular}{|l|r|r|}
\hline & \multicolumn{1}{|c|}{ Media } & Desv. típ. \\
\hline Suficiencia & 3,83 & 1,22 \\
Aprendizaje & 3,62 & 1,16 \\
Complemento & 3,63 & 1,22 \\
Amplitud conoc. & 3,47 & 1,17 \\
Conoc. empresa & 3,73 & 1,19 \\
Expectativas & 3,64 & 1,18 \\
Aumento empleabilidad & 2,54 & 1,80 \\
Valoración & 3,96 & 1,10 \\
\hline
\end{tabular}

el desempeño de las prácticas considerando estas capacidades desde tres perspectivas diferenciadas: a) la autovaloración que el alumno hace de sus propias capacidades; b) la valoración que ofrece la empresa de las capacidades demostradas por el alumno; c) la estimación que propone la empresa del perfil ideal de capacidades que debiera mostrar el alumno en el desarrollo de las prácticas.

Para este objetivo se ha realizado un contraste entre las puntuaciones obtenidas de acuerdo con estos tres puntos de vista, sobre la base de las capacidades incluidas en los cuestionarios de alumnos y empresas de forma conjunta [4]. El gráfico de los descriptivos y los resultados conseguidos son expuestos a continuación.

Gráfico 6: Contraste de valoraciones de las capacidades de los alumnos en prácticas

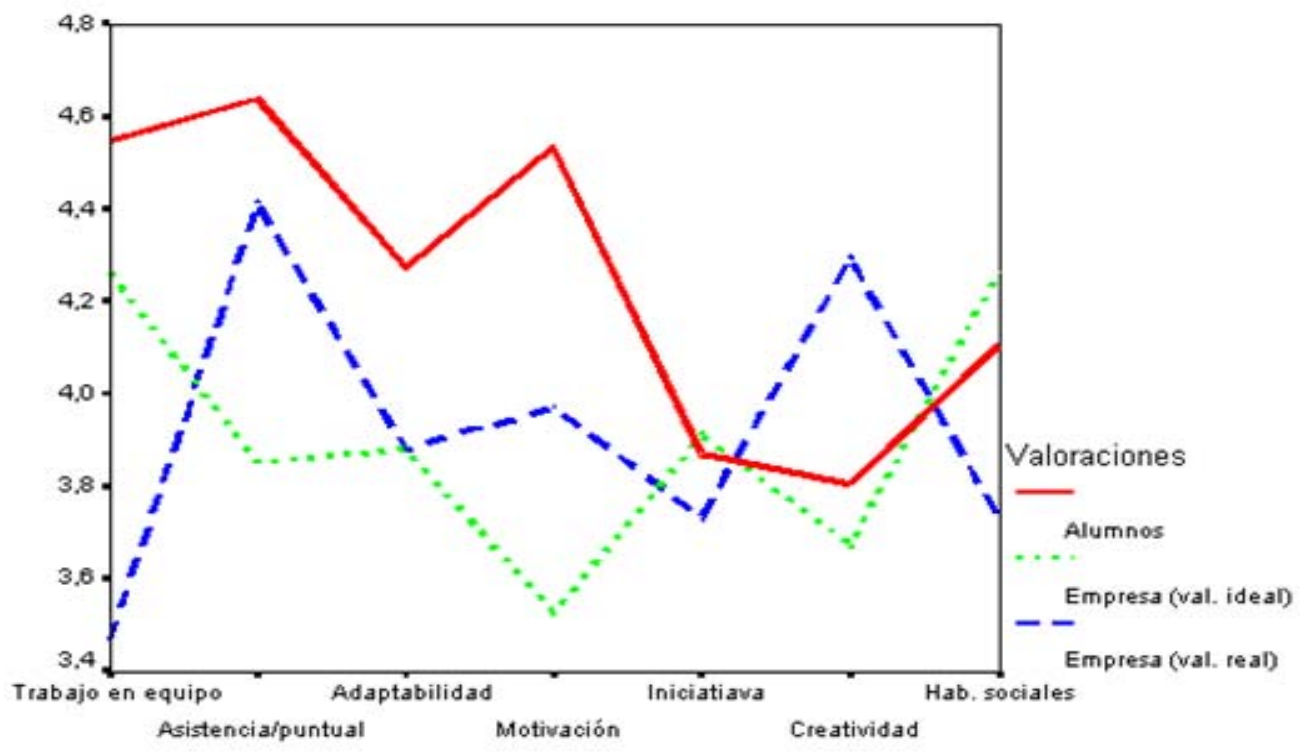


Ballesteros Velázquez, B., Manzano Soto, N. y Moriano, J. A. (2001). Seguimiento y evaluación en la UNED del sistema de prácticas de los alumnos en empresas. RELIEVE, v. 7, n. 1, p. 3-21.

http://www.uv.es/RELIEVE/v7n1/RELIEVEv7n1_1.htm

Se utilizó una escala de 1 a 5 (1 mínimo y 5 máximo) para valorar las diferentes características de los alumnos. El primer dato que podemos apreciar es que ninguna de las características obtiene una puntuación menor de 3, por tanto, todas las características de los alumnos son valoradas, al menos, como normales. Los alumnos tienden generalmente a sobre valorar sus competencias profesionales y hábitos de trabajo. De esta manera, se sienten muy competentes trabajando en equipo, relacionándose con los demás (habilidades sociales) y manifiestan una alta motivación. Sin embargo, esto contrasta con la evaluación que realizan las empresas o insti- tuciones donde los alumnos han estado realizando prácticas. Según estas últimas, los alumnos deberían ser más competentes en el trabajo en equipo y desarrollar más sus habilidades sociales; esto puede deberse a las características especiales que implica el estudio a distancia. Por otra parte, tanto la adaptación como la iniciativa que presentan nuestros alumnos resultan ideales para las empresas. Por último, encontramos muy positivo que la asistencia/puntualidad, motivación y creatividad de nuestros estudiantes superan las expectativas que tenían las empresas.

\begin{tabular}{|c|c|c|c|}
\hline \multicolumn{4}{|c|}{ Rangos } \\
\hline & Grupos & $\mathrm{N}$ & $\begin{array}{c}\text { Rango } \\
\text { promedio }\end{array}$ \\
\hline \multirow[t]{4}{*}{ Trabajo en equipo } & Alumnos & 104 & 96,44 \\
\hline & Empr.(v. ideal) & 34 & 85,26 \\
\hline & Empr. (v. real) & 34 & 57,32 \\
\hline & Total & 172 & \\
\hline \multirow[t]{4}{*}{ Asistencialpuntual. } & Alumnos & 108 & 96,35 \\
\hline & $\operatorname{Empr}_{(}(v$ ideal) & 34 & 68,71 \\
\hline & Empr. (v. real) & 34 & 83,35 \\
\hline & Total & 176 & \\
\hline \multirow[t]{4}{*}{ Adaptabilidad } & Alumnos & 104 & 89,22 \\
\hline & Empr.(v. ideal) & 34 & 75,62 \\
\hline & Empr. (v. real) & 34 & 89,07 \\
\hline & Total & 172 & \\
\hline \multirow[t]{4}{*}{ Motivación } & Alumnos & 108 & 99,09 \\
\hline & Empr.(v. ideal) & 34 & 65,50 \\
\hline & Empr. (v. real) & 34 & 77,85 \\
\hline & Total & 176 & \\
\hline \multirow[t]{4}{*}{ Iniciaativa } & Alumnos & 103 & 83,38 \\
\hline & Empr.(v. ideal) & 34 & 88,13 \\
\hline & Empr. (v. real) & 34 & 91,79 \\
\hline & Total & 171 & \\
\hline \multirow[t]{4}{*}{ Creatividad } & Alumnos & 104 & 79,88 \\
\hline & Empr.(v. ideal) & 34 & 86,66 \\
\hline & Empr. (v. real) & 34 & 106,59 \\
\hline & Total & 172 & \\
\hline \multirow[t]{4}{*}{ Hab. sociales } & Alumnos & 104 & 86,34 \\
\hline & $\operatorname{Empr}_{(}(v$ ideal) & 34 & 90,49 \\
\hline & Empr. (v. real) & 34 & 83,01 \\
\hline & Total & 172 & \\
\hline
\end{tabular}


Ballesteros Velázquez, B., Manzano Soto, N. y Moriano, J. A. (2001). Seguimiento y evaluación en la UNED del sistema de prácticas de los alumnos en empresas. RELIEVE, v. 7, n. 1, p. 3-21. http://www.uv.es/RELIEVE/v7n1/RELIEVEv7n1_1.htm

\begin{tabular}{|c|c|c|c|c|c|c|c|}
\hline \multicolumn{8}{|c|}{ Fstadístirns dp } \\
\hline & $\begin{array}{c}\text { Trabaio } \\
\text { eguir } \\
\end{array}$ & $\begin{array}{c}\text { Asistenc } \\
\text { aunitu }\end{array}$ & Adantabili & Motivaci & Iniciati & Creativid & $\begin{array}{c}\text { Hab } \\
\text { sociale }\end{array}$ \\
\hline Chi- & 19.25 & 10,69 & 2,36 & 16.11 & .90 & 8.25 & .44 \\
\hline al & 2 & 2 & 2 & 2 & 2 & 2 & 2 \\
\hline $\mathrm{Sig}$ & 00 & 00 & 30 & 00 & 63 & 01 & 80 \\
\hline
\end{tabular}

El contraste realizado pone de manifiesto la existencia de diferencias significativas entre las valoraciones emitidas para algunas variables. Así, el alumno piensa que sabe trabajar en equipo, mientras que la empresa dice que realmente no es tanta su capacidad de trabajar conjuntamente en el período de prácticas. Por otra parte, el alumno piensa que no posee una gran creatividad, mientras que la empresa afirma todo lo contrario, siendo una de las capacidades que mayormente les atribuye. Es más, en el perfil ideal de alumno la empresa quisiera incluso que el alumno fuera algo menos creativo. Este hecho plantea grandes interrogantes sobre qué es lo que buscan las empresas, mereciendo ser objeto de estudio para posteriores investigaciones en las que se disponga de mayor volumen de datos referidos a este colectivo. De todas maneras, hay que pensar que estas valoraciones están referidas a prácticas, donde sin duda el grado de complejidad de las tareas es menor que en los puestos laborales $\mathrm{y}$, por tanto, la capacidad creativa no encuentre el espacio más apropiado para manifestarse.

Debido a las características especiales que rodean los estudios a distancia, les preguntamos a las empresas si observaban alguna característica especial en los alumnos de la UNED, su respuesta ha sido afirmativa en el 50\% de los casos. Las características que las empresas destacan de nuestro alumnado se pueden resumir en los siguientes puntos:
1. Alta motivación: "ganas de trabajar, voluntad, disponibilidad, perseverancia, constancia y efectividad".

2. Buena formación: "capacidad de aprendizaje, interés por aprender y buen nivel de educación”.

3. Responsabilidad: "seriedad, alto compromiso y buena imagen”.

4. Autonomía: "autosuficiencia, autodisciplina y libertad de horario”.

\subsection{Valoración de las diferencias significati- vas en el desarrollo de prácticas según la titu- lación}

Para el estudio del objetivo que pretende conocer la existencia de diferencias en el desarrollo de las prácticas dependiendo del tipo de carrera cursada por el alumno, hemos contrastado las valoraciones emitadas por los alumnos sobre aspectos relacionados con las prácticas, tanto en su transcurso como después de finalizadas, utilizando como variable de clasificación tipos de carreras que aparecen en la tabla que sigue. Hemos prescincido de las aportaciones de alumnos pertenecientes a otros estudios, dado que el reducido tamaño de la muestra en estas otras carreras. Por otra parta, indicamos que en el área de Empresariales/Económicas se ha incluido igualmente la carrera de Administración y Dirección de Empresas. 
Ballesteros Velázquez, B., Manzano Soto, N. y Moriano, J. A. (2001). Seguimiento y evaluación en la UNED del sistema de prácticas de los alumnos en empresas. RELIEVE, v. 7, n. 1, p. 3-21.

http://www.uv.es/RELIEVE/v7n1/RELIEVEv7n1_1.htm

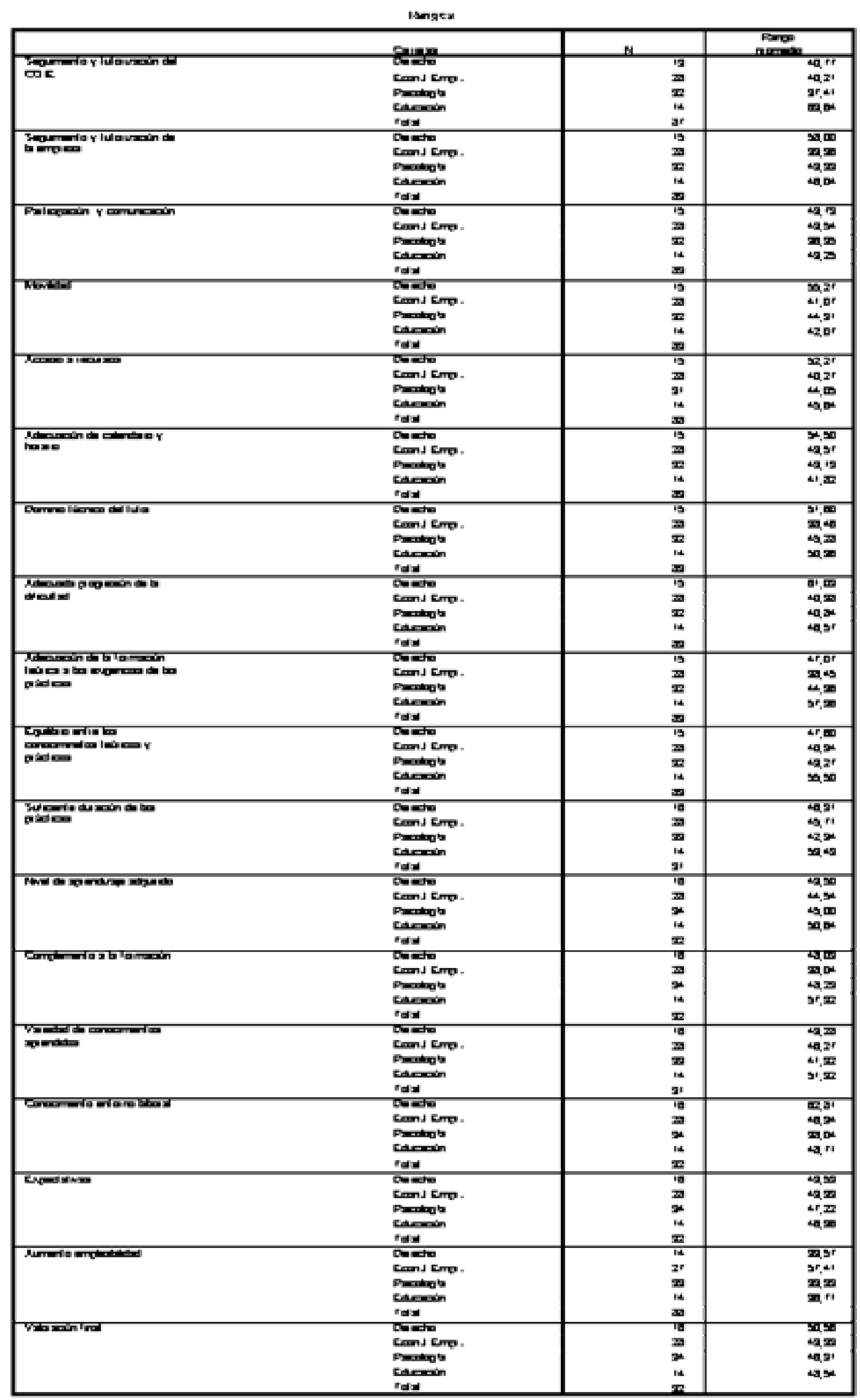


Ballesteros Velázquez, B., Manzano Soto, N. y Moriano, J. A. (2001). Seguimiento y evaluación en la UNED del sistema de prácticas de los alumnos en empresas. RELIEVE, v. 7, n. 1, p. 3-21. http://www.uv.es/RELIEVE/v7n1/RELIEVEv7n1_1.htm

Estadísticos de contraste ${ }^{\mathrm{a}, \mathrm{b}}$

\begin{tabular}{|c|c|c|c|}
\hline & Chi-cuadrado & $\mathrm{gl}$ & Sig. asintót \\
\hline Seg. COIE & 18,313 & 3 & .000 \\
\hline Seg. empresa & 5,648 & 3 & .130 \\
\hline Part/ Comunic. & 5,717 & 3 & .126 \\
\hline Mowil. & 3,411 & 3 & .332 \\
\hline Acceso & 2,365 & 3 & 500 \\
\hline Calendario/Horario & 2,853 & 3 & .415 \\
\hline Dom tếcnico tutor & 3,750 & 3 & .290 \\
\hline Progr diffcultad & 8,167 & 3 & .043 \\
\hline Ajuste formación teórica & 5,538 & 3 & .136 \\
\hline Equilibrio teoriarpráctica & 3,875 & 3 & 275 \\
\hline Duración & 1,726 & 3 & .631 \\
\hline Aprendizaje & 862 & 3 & 835 \\
\hline Compl de formación & 5,686 & 3 & 128 \\
\hline Variedad de conoc. & 1,725 & 3 & 631 \\
\hline Conoc. entomo lab. & 10,125 & 3 & 018 \\
\hline Expectativas & .547 & 3 & 908 \\
\hline Aumento empleab. & 10,529 & 3 & .015 \\
\hline Valoración final & 915 & 3 & 822 \\
\hline
\end{tabular}

Los resultados ponen de manifiesto la existencia de algunas diferencias significativas, aunque éstas no son muy numerosas. La primera de ellas, y es para el COIE uno de los aspectos más relevantes, tiene que ver con el seguimiento y tutorización de las prácticas por parte del COIE, aspecto en el cual destaca la elevada valoración de los alumnos procedentes de Educación. Este hecho se explica si consideramos que la mayor parte de los convenios establecidos para alumnos de Educación han sigo gestionados en los COIEs de Centros Asociados. Los coordinadores de estos centros proceden principalmente del ámbito de la Pedagogía y Psicología. Esta razón justifica una mayor facilidad para el establecimiento de relaciones con empresas orientadas hacia la firma de un convenio. Por otra parte, al tener los COIEs de Centros Asociados acotada la actividad a su zona territorial, evita en gran medida la masificación excesiva de alumnos, por lo que las tareas de seguimiento y tutorización pueden llevarse a cabo de forma adecuada.
Otras diferencias significativas que se señalan guardan relación con la progresión de la dificultad en el desarrollo de las prácticas, así como en la valoración sobre la oportunidad que ofrecen las prácticas mismas para el conocimiento del entorno laboral. En ambos aspectos, sobresalen los alumnos de Derecho sobre las valoraciones de los estudiantes de los otros grupos.

Un último aspecto hace referencia al aumento de empleabilidad que conlleva la realización de prácticas. En este sentido, los alumnos del ámbito de Económicas y Empresariales son los que señalan unas valoraciones más elevadas, contrastando así con los alumnos de educación que son los que menos repercusión ven en las prácticas para su acceso al mercado laboral. Hay que considerar que para esta carrera concreta existe un volumen importante de ofertas en el sector público, donde los méritos por la realización de prácticas no son valorados expresamente. 


\section{CONCLUSIONES Y PROPUES- TAS DE ACCIÓN}

Los resultados de la presente investigación nos permiten afirmar que las prácticas en empresas son consideradas como muy valiosas tanto por los alumnos como por las empresas. Los alumnos adquieren una formación práctica que les permite descubrir la aplicación de los conocimientos adquiridos durante su carrera a situaciones reales de trabajo e ir adaptando las propias actitudes a las exigencias profesionales. El estudiante se enfrenta a situaciones reales y cotidianas del mundo laboral bajo la atención de su tutor que le permiten desarrollar sus competencias profesionales, aumentar su capital social (red de contactos) y adquirir confianza en sí mismo. De esta forma, las prácticas se convierten en un eficaz medio de iniciación o socialización laboral.

Por otra parte, las prácticas se han revelado como un excelente apoyo para la inserción laboral para los titulados universitarios. De forma indirecta, las prácticas son especialmente valoradas en los procesos de selección porque aportan un doble valor al currículum del titulado universitario. Por un lado, garantizan en el aspirante cierto conocimiento práctico del entorno de trabajo y de las competencias profesionales, por otro permiten deducir que se trata de una persona con inquietudes profesionales que toma la iniciativa de llevar su formación más allá de los estrictamente obligatorio para la obtención del título. De forma directa, las prácticas son en sí mismas un canal para acceder al primer empleo. Según los resultados de este estudio, uno de cada tres alumnos que realizan prácticas en empresas es contratado a la finalización de las mismas. Por tanto, las prácticas no tienen sólo un carácter formativo, sino que suponen una auténtica pasarela entre el mundo académico y el primer empleo.

Hemos señalado los aspectos positivos de las prácticas pero también hay ciertos peligros que el alumno debe tener en cuenta: a) Algunas empresas utilizan a los alumnos en prácticas como fuente de mano de obra barata y en algunos casos gratuita. El alumno debe valorar su contribución a la empresa y las recompensas obtenidas (formación) y no olvidar que el objetivo principal es aprender y si este queda desvirtuado debe abandonarlas y ponerlo en conocimiento del COIE.

b) En algunas organizaciones quizá no se disponga de una buena planificación de las prácticas o pueden que haya problemas de distinta índole; es en estos casos donde el alumno debe poner con mayor realce su iniciativa y su voluntad de aprender y autogestionar si cabe su aprendizaje.

c) El alumno debe valorar si la dedicación a las prácticas le está perjudicando en su rendimiento académico. Debe administrar adecuadamente su tiempo.

A continuación se ofrecen algunas sugerencias o modificaciones propuestas por los alumnos y las empresas para la mejora de las prácticas.

\section{Alumnos}

- En relación con la planificación de las prácticas:

- Especificación por parte de la empresa de las actividades que se habrán de realizarse en el transcurso de las prácticas.

- Propuesta de actividades relacionadas con el perfil formativo del alumno.

- Compatibilidad horaria con el estudio del alumno, aprovechando para ello períodos de vacaciones y, en cualquier caso, respetando las jornadas de exámenes.

- En relación con la duración de las prácticas:

- Diversidad de opiniones sobre la valoración del número total de horas, dependiendo en gran medida del tipo de prácticas y duración de las mismas. No obstante, existe un acuerdo notable en la estimación de 500 horas como período suficiente 
para adquirir una experiencia inicial con el mundo de la empresa, compatible con los estudios.

- Se subraya la idea de aprovechamiento de las tareas por encima de la extensión más o menos prolongada del período de prácticas.

\section{Empresas}

- En relación con la planificación de las prácticas:

- Antes de la selección de los alumnos se podrían organizar visitas a las empresas para recibir mayor información, así como organizar de seminarios y jornadas dentro de la universidad donde las empresas dieran a conocer sus propias características y los puestos que ofrecen en prácticas.

- Realización de un documento básico entre el COIE y la empresa donde se estructure y planifique el desarrollo de la práctica.

\section{BIBLIOGRAFÍA}

Aragón, R.N. y otros (1999). La imagen social de la universidad. En AIDIPE: Nuevas realidades educativas, nuevas necesidades metodológicas. Málaga: CEDMA.

Ausín, T. y otros (1997). Análisis de necesidades de orientación en alumnos universitarios. En Actas del Congreso de Orientación Universitaria y Evaluación de la Calidad. Bilbao: Universidad del País Vasco.

Ballesteros, B., Guillamón, J.R., Manzano, N., Moriano, J.A. y Palací, F. (2001). Técnicas de inserción laboral. Guía universitaria para la búsqueda de empleo. Madrid: UNED. (En prensa).

Cajide, J. y otros (1997). La reforma de las titulaciones universitarias de Física, Farmacia, Agrónomos y Agroalimentaria. Un estudio comparativo. En Actas del VIII Congreso Nacional de Modelos de investigación Educativa. Sevilla: AIDIPE/ICE de la Universidad de Sevilla.

Casanova Arias, P.F.; Moral Vico, A. (1989). La evaluación de las prácticas de enseñanza en los centros de EGB. Revista Interuniversitaria de Formación del Profesorado, 6, 85-89

Escudero Escorza, T. et al.(2000). Evaluación de las prácticas en la licenciatura de Medicina. Zaragoza: Instituto de Ciencias de la Educación.

Estela, M.J. y Escartin, M.J. (1988). La evaluación de prácticas en una escuela de trabajo social. Revista de trabajo social, 0109, 03, 162-171

Fuentes, M.; Machin, J.; Muñoz, G.; Pardillos, G.; Redon, F.; Sancho, J.M.; Sanchon, I.; Serrano, C.; Tarin, T.; Zaragoza, P. (1990). Evaluación de las prácticas organizativas de los centros. Cuadernos de Pedagogía, 177, 54-57.

González Ramírez, MªT. (2000) (Coord.). Evaluación y gestión de la calidad educativa. Un enfoque metodológico. Málaga: Aljibe.

Jiménez Jiménez, B. y Zurita Morales, F.J. (1996). Evaluación de las prácticas en alternancia "dual" de las de FP reglada. Universitas tarraconensis. Revista de ciencies de l'educacio, II, 181-187.

Lobato, C. (Ed.) (1996). Desarrollo profesional y prácticum en la Universidad.

López Rupérez, F. (2000). Gestión de calidad y mejora escolar. En R. Pérez Juste, F. López Rupérez, $M^{\mathrm{a}} \mathrm{D}$. Peralta y P. Municio. Hacia una educación de calidad. Gestión, instrumentos y evaluación, (pp. 45-73). Madrid: Narcea.

Sánchez García, M.F. (1999). Necesidades y servicios de orientación universitaria en la Comunidadd de Madrid. Madrid: UNED.

Sebastián, A. y otros (1996). Análisis de las necesidades de información y orientación en los alumnos de la UNED. (Inédito).

Segura Egea, J.J. y Jiménez RubioManzanares (1998). Propuesta metodológica para la evaluación de las prácticas clínicas de odontología. Revista de enseñanza universitaria, 12, 11-20.

UNED (2000). Guía de evaluación. (Documento de uso interno). 
Ballesteros Velázquez, B., Manzano Soto, N. y Moriano, J. A. (2001). Seguimiento y evaluación en la UNED del sistema de prácticas de los alumnos en empresas. RELIEVE, v. 7, n. 1, p. 3-21.

http://www.uv.es/RELIEVE/v7n1/RELIEVEv7n1_1.htm

\section{NOTAS}

[1] En suma, el equipo de investigación quedó integrado por COIE-Sede Central, C.A. Talavera, C.A. Cartagena, C.A. Valencia, C.A. Rioja, C.A. Denia.

[2] Hay que considerar el hecho de que una misma empresa suscribe un convenio con la UNED en el cual pueden participar un grupo de alumnos. Esta razón explica el desequilibrio aparente entre número de empresas y número de alumnos.
[3] El artículo sexto del Real Decreto 1497/1981 establece que "el convenio podrá prever la aportación por las Empresas de una cantidad en concepto de bolsa o ayuda al estudio, que será satisfecha en la forma que determine el propio convenio".

[4] El cuestionario de alumnos ofrece un repertorio más extenso sobre capacidades del alumno en prácticas. No obstante, se ha tenido que reducir obviamente, busacando la coincidencia de elementos entre el cuestionario de alumnos y empresas.

\section{ARTICLE RECORD / FICHA DEL ARTÍCULO}

\begin{tabular}{|c|c|}
\hline $\begin{array}{l}\text { Reference / } \\
\text { Referencia }\end{array}$ & $\begin{array}{l}\text { Ballesteros Veláquez, Belén, Manzano Soto, Nuria y Moriano, Juan Antonio (2001). Seguimiento } \\
\text { y evaluación en la UNED del sistema de prácticas de los alumnos en empresas. RELIEVE, vol. } 7 \text {, } \\
\text { n. 1. Consultado en www.uv.es/RELIEVE/v7n1/RELIEVEv7n1_1.htm en (poner fecha). }\end{array}$ \\
\hline $\begin{array}{l}\text { Title / } \\
\text { Título }\end{array}$ & $\begin{array}{l}\text { Segumiento y evaluación en la UNED del sistema de prácticas de los alumnos en empresas [Su- } \\
\text { pervision and evaluation of business internships for students in the UNED] }\end{array}$ \\
\hline Authors / Autores & Belén Ballesteros Velázquez, Nuria Manzano Soto y Juan Antonio Moriano \\
\hline Review / Revista & Revista ELectrónica de Investigación y EValuación Educativa (RELIEVE), v. 7, n.1 \\
\hline ISSN & $1134-4032$ \\
\hline Abstract / & $\begin{array}{l}\text { In this article we present the basic guidelines proposed for the Guidance, Information and Em- } \\
\text { ployment Center (COIE) of the National University for Distance Education (UNED) for the de- } \\
\text { velopment, management, and evaluation of educational co-operation contracts for field experi- } \\
\text { ence in businesses, in which students are requesting to participate with greater frequency. The } \\
\text { beginning of this research focuses on the supervision of the development of these programs, and } \\
\text { establishing guidelines and design procedures in order to follow-up to improve them. The con- } \\
\text { clusions presented here are drawn from results that were obtained in the evaluation of intern- } \\
\text { ships during the academic course 1998/99 and 1999/00, according to the perspective of the par- } \\
\text { ticipating enterprises observing student interns, and are organized into a proposal. }\end{array}$ \\
\hline Resumen & $\begin{array}{l}\text { En este artículo se presentan las líneas básicas de trabajo iniciadas por el Centro de Orientación, } \\
\text { Información y Empleo (COIE) de la UNED para la evaluación de uno de los ámbitos de activi- } \\
\text { dad que resulta cada vez más solicitado por los alumnos: la gestión de convenios de cooperación } \\
\text { educativa para la realización de prácticas en empresas. La necesidad de supervisar su desarrollo, } \\
\text { establecer mecanismos de seguimiento y, en definitiva, diseñar procedimientos para su mejora, } \\
\text { constituyen el punto de partida de esta investigación. Se presentan los resultados conseguidos en } \\
\text { la evaluación de prácticas, tanto desde el punto de vista de las empresas como de los alumnos, } \\
\text { que han tenido lugar en los cursos académicos 1998/99 y 1999/00, junto con las conclusiones } \\
\text { obtenidas y medidas de mejora propuestas. }\end{array}$ \\
\hline $\begin{array}{l}\text { Keywords / } \\
\text { Descriptores }\end{array}$ & $\begin{array}{l}\text { Evaluation, supervision, practical training, internship } \\
\text { Evaluación, seguimiento, prácticas. }\end{array}$ \\
\hline $\begin{array}{l}\text { Institution / } \\
\text { Institución }\end{array}$ & Universidad Nacional de Educación a Distancia (UNED, España) \\
\hline $\begin{array}{l}\text { Publication site / } \\
\text { Dirección }\end{array}$ & http://www.uv.es/RELIEVE \\
\hline $\begin{array}{l}\text { Language / } \\
\text { Idioma }\end{array}$ & Spanish (Title, abstract and keywords in English ) \\
\hline
\end{tabular}


Ballesteros Velázquez, B., Manzano Soto, N. y Moriano, J. A. (2001). Seguimiento y evaluación en la UNED del sistema de prácticas de los alumnos en empresas. RELIEVE, v. 7, n. 1, p. 3-21.

http://www.uv.es/RELIEVE/v7n1/RELIEVEv7n1_1.htm

\section{Revista ELectrónica de Investigación y EValuación Educativa (RELIEVE)}

\section{[ ISSN: 1134-4032 ]}

(C) Copyright, RELIEVE. Reproduction and distribution of this articles it is authorized if the content is no modified and their origin is indicated (RELIEVE Journal, volume, number and electronic address of the document).

(C) Copyright, RELIEVE. Se autoriza la reproducción y distribución de este artículo siempre que no se modifique el contenido y se indique su origen (RELIEVE, volumen, número y dirección electrónica del documento). 